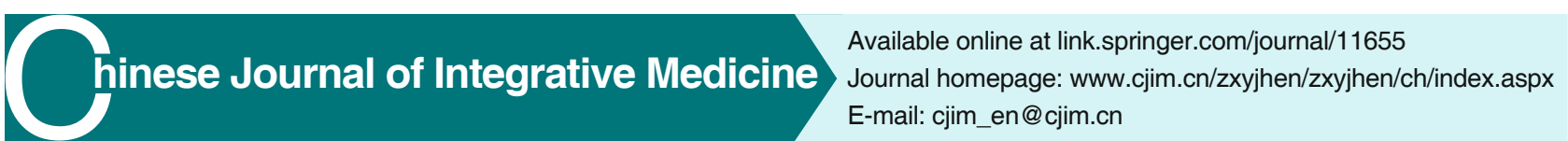

Original Article

\title{
Medication Rule Analysis of the Diagnosis and Treatment Programs of Chinese Medicine for the Prevention and Treatment of COVID-19 in China*
}

\author{
JI Xin-yu', MA Yan ${ }^{1}$, SHI Shuai', LIU Si-hong ${ }^{3}$, TONG Lin ${ }^{3}$, LYU Cheng ${ }^{1}$, ZHANG Hua-min ${ }^{4}$, and WANG Yan-ping ${ }^{1}$
}

\begin{abstract}
Objectives: To summarize the measures and rules of Chinese medicine (CM) and provide reference for clinical application in the prevention and treatment of coronavirus disease (COVID-19). Methods: The data source was from CM COVID-19 prevention and treatment programs on government websites and official media websites of the different provinces and cities. The search lasted from December 8, 2019 to March 10, 2020. Main variables were medication frequency and combinations of medicines. Cluster analysis and complex network analysis were used by prevention and treatment stage and by area. Results: Among $27 \mathrm{CM}$ diagnosis and treatment plans, 203 therapeutic prescriptions were enrolled, of which the top 4 herbs were: Radix glycyrrhizae, Semen armeniacae amarum, Herba ephedrae, and Herba agastachis, respectively. The core combinations were Herba ephedrae and Semen armeniacae amarum. Forty-eight preventive formulae were identified. Ten herbs, including Radix Astragali seu hedysari, Radix glycyrrhizae, Radix saposhnikoviae, Flos lonicerae, etc. were most frequently used. The core prescription of CM compatibility was Radix astragali seu hedysari, Radix glycyrrhizae, and Radix saposhnikoviae, which is the main component of Yu Ping Feng San. There were 45 prevention and treatment prescriptions in East China; the most used CM was Radix glycyrrhizae, Herba agastachis, Pericarpium citri reticulatae, and Gypsum fibrosum. Fifty prescriptions were identified in North China. According to CM analyses, Herba agastachis, Semen armeniacae amarum, Herba ephedrae, and Poria were most frequently used. Conclusions: CM for COVID-19 prevention mainly focuses on improving human immunity; for treatment, prescription focuses on clearing the lungs and removing dampness. Prescriptions vary with regions, perhaps due to climatic and environmental differences, which help clinicians to quickly make $\mathrm{CM}$ plans and treat patients according to clinical status, further minimizing resource wastage.
\end{abstract}

KEYWORDS COVID-19, Chinese medicine, regularity of medication

In December 2019, corona virus disease 2019 (COVID-19) was broken out, which was mainly characterized by the symptoms of fever, myalgia, fatigue, and cough. ${ }^{(1)}$ Shortly after that, cases of the infection were also reported in the US, Japan, South Korea, and 24 other countries around the world. ${ }^{(2)}$ As of March 24, 2020, COVID-19 had affected 334,981 people in 190 countries, causing 14,652 deaths. ${ }^{(3)}$ Unfortunately, no specific antiviral medicines are currently available for this disease. ${ }^{(4)}$ Hence, the prevention and treatment of this infection have made clinical research challenging. However, a series of clinical practice results have demonstrated that Chinese medicine (CM) plays a significant role in prevention and treatment of epidemic diseases, ${ }^{(5)}$ which could shorten the disease course ${ }^{(6)}$ and reduce the mortality rate ${ }^{(7)}$ side effects and complications of COVID-19. ${ }^{(8)}$
After the outbreak of COVID-19, China has formulated been updating its national diagnostic and therapeutic guidelines for COVID-19. CM is also

CThe Chinese Journal of Integrated Traditional and Western Medicine Press and Springer-Verlag GmbH Germany, part of Springer Nature 2021

*Supported by National Science and Technology Major Project (Nos. 2018ZX10101001-005-003, 2018ZX10101001-005-004), the National Key Research and Development Project (Nos. 2018YFC1704401, 2019YFC1709802)

1. Institute of Basic Research in Clinical Medicine, China Academy of Chinese Medical Sciences, Beijing (100700), China; 2. Guang'anmen Hospital, China Academy of Chinese Medical Sciences, Beijing (100053), China; 3. Institute of Information on Traditional Chinese Medicine, China Academy of Chinese Medical Sciences, Beijing (100700), China; 4. Institute of Chinese Materia Medica, China Academy of Chinese Medical Sciences, Beijing (100700), China

Correspondence to: Prof. WANG Yan-ping, E-mail: wangyanping4816@163.com

DOI: https://doi.org/10.1007/s11655-021-2880-1 
recommended for use in prevention and treatment programs. Moreover, given the local climate, location, and other factors, different provinces and cities have formulated different prevention and treatment plans of $\mathrm{CM}$ based on the national guidelines for COVID-19. ${ }^{(9)}$ Particularly, among the confirmed cases, $91.5 \%$ of the patients used $\mathrm{CM}$, with a total efficiency rate of more than $90 \% .^{(10)}$ In the Wuhan Jiangxia Mobile Cabin Hospital, patients not only took CM also practiced Tai chi and Baduanjin. ${ }^{(11)}$ Moreover, typical CM therapy, such as massage and moxibustion, is widely used for COVID-19 patients. In order to enhance the understanding of COVID-19 by CM, it is urgent and important to actively impore the diagnosis and treatment programs in China. Therefore, we analyze and summarize the medication rules of $\mathrm{CM}$ for prevention and treatment of COVID-19, rules of CM in different regions of China, which provide reference for clinical application of $\mathrm{CM}$ in the treatment of COVID-19.

\section{METHODS}

\section{Data Sources}

The data source was from CM COVID-19 prevention and treatment programs on government websites or official media websites in China. The keywords were "COVID-19", "CM", "diagnosis and treatment plan", and "prevention and treatment plan". The search was conducted from December 8, 2019 to March 10, 2020.

\section{Inclusion and Exclusion Criteria}

Inclusion criteria: (1) CM diagnosis and treatment plan; (2) prevention and treatment prescriptions for adults and children; (3) treatment and prevention prescriptions recommended by the health committees of all provinces, cities, and autonomous regions.

Exclusion criteria: (1) recommended Chinese patent medicine; (2) prescriptions of ethnic medicines with local characteristics.

\section{Data Collection and Analysis}

A total of 48 prevention prescriptions and 203 treatment prescriptions from 27 provinces and cities were included. Two authors independently entered the data. Meanwhile, according to "Pharmacopoeia of the People's Republic of China",(12) "Dictionary of Traditional Chinese Medicine", ${ }^{(13)}$ and "Chinese Medicine", ${ }^{(14)}$ the names of CM were standardized.
Through the "medication statistics" function in the "data mining and analysis" module in the "ancient and modern medical case platform V2.2.1" (Institute of Information on Traditional Chinese Medicine, China Academy of Chinese Medical Sciences), data mining and statistical analysis were performed to evaluate the frequency and compatibility of four properties and five tastes of $\mathrm{CM}$ and meridian tropism of $\mathrm{CM}$. The clustering analysis and complex network analysis in the "multi-dimensional analysis" module were used to analyze the prescription rules in different regions and explore the medication rules.

\section{RESULTS}

\section{Frequency of CM in Different Periods}

From the 27 diagnosis and treatment programs, there were 203 prescriptions for the treatment of COVID-19 with CM, with 262 kinds of CM, totaling 2,199 times. The top 4 kinds of $\mathrm{CM}$ used in the prescriptions were Radix glycyrrhizae (Gancao), Semen armeniacae amarum (Xingren), Herba ephedrae (Mahuang), and Herba agastschis (Huoxiang). Forty-eight prescriptions were issued for the prevention of COVID-19 with CM, with 89 kinds of CM, totaling 346 times. The frequency of the treatment and prevention with $\mathrm{CM}$ in the top 10 are shown in Figures 1 and 2, respectively.

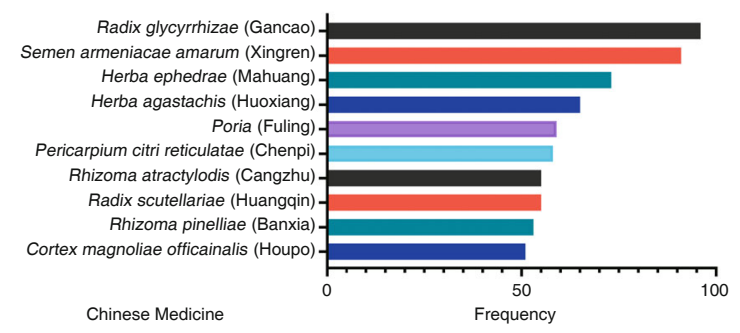

Figure 1. Chinese Medicine for Treatment of COVID-19 (Top 10)

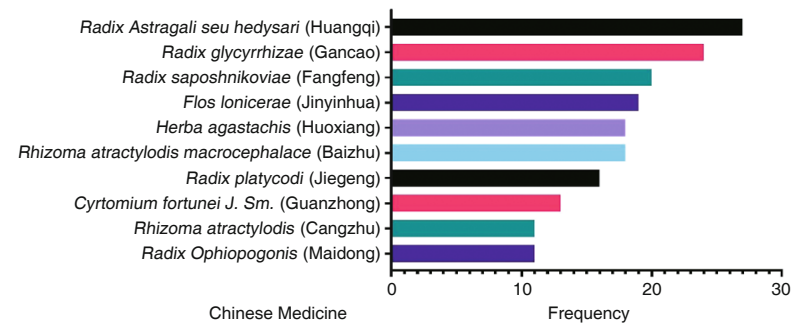

Figure 2. Chinese Medicine for Prevention of COVID-19 (Top 10)

\section{Compatibility of CM in Different Periods}

The compatibility of $\mathrm{CM}$ in the prevention and treatment stage is shown in Tables 1 and 2, respectively, which indicates the top 10 compatibilities of CM with 
the degree of confidence $\geqslant 0.7$. The core compatibility of treatment medicine was Herba ephedrae (Mahuang), Semen armeniacae amarum (Xingren), and Radix scutellariae (Huangqin), while the core compatibility of preventive medicine was Radix saposhnikoviae (Fangfeng), Radix astragali seu hedysari (Huangqi), and Rhizoma atractylodis macrocephalace (Baizhu).

Table 1. Compatibility of the Top 10 Chinese Medicines for Treatment of COVID-19

\begin{tabular}{|c|c|c|c|c|}
\hline No. & $\mathrm{CM}$ & $\begin{array}{l}\text { Co-occurrence } \\
\text { frequency }\end{array}$ & Confidence & Support \\
\hline 1 & $\begin{array}{l}\text { Herba ephedrae- } \\
\text { Semen armeniacae amarum } \\
\text { (Mahuang-Xingren) }\end{array}$ & 60 & 0.82 & 0.3 \\
\hline 2 & $\begin{array}{l}\text { Radix scutellariae- } \\
\text { Semen armeniacae amarum } \\
\text { (Huangqin-Xingren) }\end{array}$ & 40 & 0.73 & 0.2 \\
\hline 3 & $\begin{array}{l}\text { Rhizoma pinelliae- } \\
\text { Poria (Banxia-Fuling) }\end{array}$ & 39 & 0.74 & 0.19 \\
\hline 4 & $\begin{array}{l}\text { Gypsum fibrosum- } \\
\text { Semen armeniacae amarum } \\
\text { (Shigao-Xingren) }\end{array}$ & 36 & 0.73 & 0.18 \\
\hline 5 & $\begin{array}{l}\text { Cortex magnoliae officinalis- } \\
\text { Herba Agastachis } \\
\text { (Houpo-Huoxiang) }\end{array}$ & 36 & 0.71 & 0.18 \\
\hline 6 & $\begin{array}{l}\text { Gypsum fibrosum- } \\
\text { Herba ephedrae } \\
\text { (Shigao-Mahuang) }\end{array}$ & 35 & 0.71 & 0.17 \\
\hline 7 & $\begin{array}{l}\text { Fructus tsaoko-Herba ephedrae } \\
\text { (Caoguo-Mahuang) }\end{array}$ & 33 & 0.85 & 0.16 \\
\hline 8 & $\begin{array}{l}\text { Semen lepidii seu descurainiae- } \\
\text { Semen armeniacae amarum } \\
\text { (Tinglizi-Xingren) }\end{array}$ & 32 & 0.8 & 0.16 \\
\hline 9 & $\begin{array}{l}\text { Fructus tsaoko- } \\
\text { Rhizoma atractylodis } \\
\text { (Caoguo-Cangzhu) }\end{array}$ & 31 & 0.79 & 0.15 \\
\hline 10 & $\begin{array}{l}\text { Semen lepidii seu descurainiae- } \\
\text { Herba ephedrae } \\
\text { (Tinglizi-Mahuang) }\end{array}$ & 30 & 0.75 & 0.15 \\
\hline
\end{tabular}

\section{Cluster Analysis of CM in Different Periods}

Cluster analysis was performed for CM prescriptions in the prophylactic and therapeutic periods. European distance was selected as the distance type and the longest clustering method was selected as the clustering method as shown in Figures 3 and 4.

\section{Frequency of CM in Different Regions}

Of the $50 \mathrm{CM}$ prescriptions for the prevention and treatment of COVID-19 in North China, there were 110 kinds of $\mathrm{CM}$, totaling 444 times. The top 4 kinds of CM used in the prescriptions were Herba agastachis (Huoxiang), Semen armeniacae amarum (Xingren), Herba ephedrae (Mahuang), and Poria (Fuling). In East China, the top 4 Chinese medicine in the prescriptions were Radix glycyrrhizae (Gancao), Herba agastachis (Huoxiang), Pericarpium citri
Table 2. Compatibility of the Top 10 Chinese Medicines for the Prevention of COVID-19

\begin{tabular}{|c|c|c|c|c|}
\hline No. & $\mathrm{CM}$ & $\begin{array}{l}\text { Co-occurrence } \\
\text { frequency }\end{array}$ & Confidence & Support \\
\hline 1 & $\begin{array}{l}\text { Radix saposhnikoviae- } \\
\text { Radix astragali seu hedysari } \\
\text { (Fangfeng--Huangqi) }\end{array}$ & 18 & 0.9 & 0.38 \\
\hline 2 & $\begin{array}{l}\text { Radix saposhnikoviae- } \\
\text { Rhizoma atractylodis } \\
\text { Macrocephalace } \\
\text { (Fangfeng-Baizhu) }\end{array}$ & 16 & 0.8 & 0.33 \\
\hline 3 & $\begin{array}{l}\text { Rhizoma atractylodis } \\
\text { macrocephalace- } \\
\text { Radix saposhnikoviae } \\
\text { (Baizhu-Fangfeng) }\end{array}$ & 16 & 0.89 & 0.33 \\
\hline 4 & $\begin{array}{l}\text { Rhizoma atractylodis } \\
\text { macrocephalace-Radix astragali } \\
\text { seu hedysari (Baizhu-Huangqi) }\end{array}$ & 16 & 0.89 & 0.33 \\
\hline 5 & $\begin{array}{l}\text { Radix saposhnikoviae-- } \\
\text { Radix glycyrrhizae } \\
\text { (Fangfeng-Gancao) }\end{array}$ & 15 & 0.75 & 0.31 \\
\hline 6 & $\begin{array}{l}\text { Radix platycodi-Radix glycyrrhizae } \\
\text { (Jiegeng-Gancao) }\end{array}$ & 14 & 0.88 & 0.29 \\
\hline 7 & $\begin{array}{l}\text { Rhizoma atractylodis- } \\
\text { Radix astragali seu hedysari } \\
\text { (Cangzhu-Huangqi) }\end{array}$ & 8 & 0.73 & 0.17 \\
\hline 8 & $\begin{array}{l}\text { Fructus forsythiae-Flos lonicerae } \\
\text { (Lianqiao-Jinyinhua) }\end{array}$ & 7 & 0.7 & 0.15 \\
\hline 9 & $\begin{array}{l}\text { Perillae folium-Radix astragali seu } \\
\text { hedysari (Zisuye-Huangqi) }\end{array}$ & 4 & 1 & 0.08 \\
\hline 10 & $\begin{array}{l}\text { Dendranthema morifolium- } \\
\text { Radix Ophiopogonis } \\
\text { (Juhua-Maidong) }\end{array}$ & 4 & 0.8 & 0.08 \\
\hline
\end{tabular}

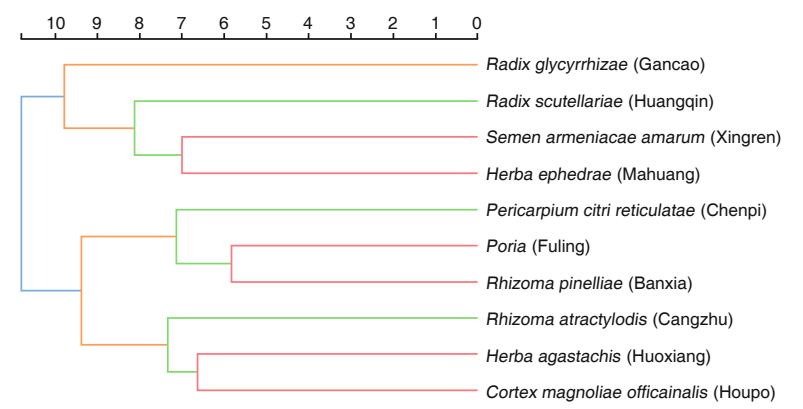

Figure 3. Clustering Analysis of Chinese Medicine for Treatment of COVID-19 (Top 10)

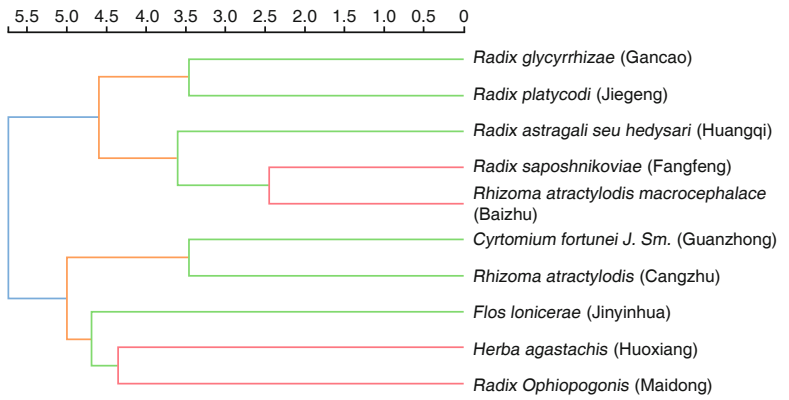

Figure 4. Clustering Analysis of Chinese Medicine for Prevention of COVID-19 (Top 10)

Reticulatae (Chenpi), and Gypsum fibrosum (Shigao) (Appendixes 1 and 2). 


\section{Compatibility of CM in Different Regions}

The compatibility of CM in North and East China was shown in supplementary materials (Appendixes 3 and 4). The tables showed a co-occurrence frequency $\geqslant 6$ and confidence $\geqslant 0.75$ in North China and a co-occurrence frequency $\geqslant 7$ and confidence $\geqslant 0.73$ in East China.

\section{Cluster Analysis in Different Regions}

Cluster analysis was used for high-frequency medicines in the prescriptions for the prevention and treatment of COVID-19 in both North and East China. European distance was selected as the distance type and the longest clustering method was chosen, as shown in supplementary materials (Appendixes 5 and 6 ).

\section{Complex Network Analysis in Different Regions}

In the data mining analysis, the complex network analysis was selected, the core medicine combination in the prescription of $\mathrm{CM}$ for prevention and treatment of COVID-19 in North and East China was extracted, and the core medicine network diagram drawn, as shown in supplementary materials (Appendixes 7 and 8 ).

\section{DISCUSSION}

COVID-19 is an acute respiratory infectious disease caused by the novel coronavirus. Fever, dry cough, and fatigue are its main symptoms. ${ }^{(15)} \mathrm{CM}$ experts used the $\mathrm{CM}$ theory to determine the etiology and pathogenesis of COVID-19. Previous studies observed that the occurrence of COVID-19 was due to the sudden cooling of the local weather in Wuhan, coupled with continuous rainfall, resulting in the surge of epidemic virus. ${ }^{(16-19)}$

The diagnosis and treatment programs of 27 provinces, municipalities, and autonomous regions all contain two periods including prevention and treatment. Therefore, we analyzed the frequency, compatibility, and clustering of $\mathrm{CM}$ in these two periods. Through frequency analysis, we found that the medicines with the highest frequency of use in CM treatment of COVID-19 were Radix glycyrrhizae (Gancao), Semen armeniacae amarum (Xingren), Herba ephedrae (Mahuang), and Herba agastachis (Huoxiang). From these frequently used $\mathrm{CM}$, it is not difficult to deduce that the treatment is mainly based on medicines that dissipate cold and dampness, while the prevention is mainly based on promotion of positive qi. Radix glycyrrhizae (Gancao), its active ingredient glycyrrhizin could potently inhibit replication of clinical isolates of severe acute respiratory syndrome coronavirus 2 (SARS-CoV-2). ${ }^{(20)}$ Moreover, Semen armeniacae amarum (Xingren), with property of relieving cough and asthma, and its active ingredient, amygdalin, can inhibit pulmonary fibrosis. ${ }^{(21)}$ Herba agastachis (Huoxiang) could remove cold and dampness and dry up Spleen. The published literature has demonstrated that it has antiviral effects, ${ }^{(22)}$ great benefits for respiratory diseases, ${ }^{(23)}$ and inhibits influenza virus. ${ }^{(24)}$ With respect to the compatibility of $\mathrm{CM}$, we also found that the treatment mainly depended on ephedra-almond-gypsum compatibility, which is the main component of Maxing Shigan Decoction (麻杏石甘汤). A randomized trial showed that Maxing Shigan Decoction with Yinqiao San (银翅散) might be used as an alternative treatment for H1N1 influenza virus infection, which could reduce the time required for fever resolution. ${ }^{(25)}$

Chinese herbs with the highest frequency of use in the prevention period were Radix glycyrrhizae (Gancao), Radix astragali seu hedysari (Huangqi), Rhizoma atractylodis macrocephalace (Baizhu), Flos lonicerae (Jinyinhua), etc. A growing number of pharmacological studies have reported that Radix astragali seu hedysar (Huangqi) can increase telomerase activity and have anti-inflammatory, immune-regulatory, hypolipidemic, antihyperglycemic, hepatoprotective, and anti-aging effects. ${ }^{(26)}$ In addition, Flos lonicerae (Jinyinhua), with the effect of clearing heat and toxicity, was the most popular herb used in the treatment of SARS and influenza A in 2003 and $2009{ }^{(27)}$ Considering compatibility and clustering, the main components of preventive prescriptions are Radix glycyrrhizae (Gancao), Radix astragali seu hedysari (Huangqi), Rhizoma atractylodis macrocephalace (Baizhu), and Radix saposhnikoviae (Fangfeng), which are the main components of Yupingfeng San (玉屏风 散), which has been used in antiviral or anti-bacterial therapy. It has been reported to prevent viral infections including SARS and enhance cellular immunity. ${ }^{(28)}$

The thought of "treatment in accordance with local conditions" is an important principle and theoretical feature of $\mathrm{CM}^{\left({ }^{(29)}\right.}$ Apart from the seasonal climate and patient's physical strength, the treatment of epidemic diseases is closely related to geographical environment and eating habits. ${ }^{(30)}$ For example, epidemic encephalitis B and SARS all show different pathogenetic characteristics in different regions and also show diversity in medication. ${ }^{(31,32)}$ And there are different $\mathrm{CM}$ diagnosis and treatment programs for COVID-19 in different regions. Therefore, 
taking East and North China as examples, we analyzed the characteristics of medicine use in the two regions.

The climate is cold and dry in winter in North China. ${ }^{(15)}$ However, due to the large-scale application of heating, the indoor and outdoor temperatures vary greatly. The degree of dryness in North China is extremely uneven throughout the year. ${ }^{(16)}$ Geographically, East China has marine climate characteristics, belonging to subtropical monsoon climate. Precipitation is abundant and tends to increase. ${ }^{(33)}$ According to this climate characteristic, "dampness" is a pathological factor in East China.

According to the Beijing diagnosis and treatment plan of CM, some patients with COVID-19 are prone to symptoms such as high-grade fever, asthma, palpitation, and delirium. Tianjin CM guidance demonstrated that the basic pathogenesis in Tianjin area is characterized by dampness, heat, toxin, and blood stasis. In other words, from the perspective of $\mathrm{CM}$, both regions believe that the etiology of COVID-19 is linked to the characteristics of heat and dryness. Also, the same viewpoint is shown in the Inner Mongolia treatment program. Therefore, it is not difficult to find that in North China, many diagnosis and treatment schemes highlight the characteristics of "heat" and "dryness". However, "Dampness" is the pathological factor in the diagnosis and treatment plans in East China. For example, the treatment plan of Shanghai, Anhui Province, highlights the pathogenetic characteristic of "dampness" as the main feature, which is different from North China.

Through the analysis of frequency and compatibility of $\mathrm{CM}$, cold and dampness-removing medicines were found in the high-frequency CM used in East and North China, such as Herba agastachis (Huoxiang), Radix glycyrrhizae (Gancao), and Semen armeniacae amarum (Xingren). In East China, dampness-dispelling medicines are the top 10 frequently used CM, while Radix scutellariae (Huangqin) and Flos lonicerae (Jinyinhua) are more frequently used in North China. It is worth noting that Alpinia katsumadai hayata (Caodoukou) is more frequently used in combination in East China but less frequently in North China. It has the effect of drying dampness, promoting qi and warming to stop nausea, whose active ingredient, alpinetin, markedly inhibits tumor necrosis factor- $\alpha$, interleukin-6, and $1 \beta$ both in vitro and in vivo. In North China, many antipyretics are used, and the frequency of use is higher than that in East China. According to the compatibility characteristics of $\mathrm{CM}$, the combination of cold-removing dampness medicine and heat-clearing medicine are both adopted in East and North China. Through cluster analysis, the heat-clearing medicines and qi-invigorating medicine are the main medicines in 6 categories of CM in North China, while dampness-dispelling medicine are the main medicines in East China. Through complex network analysis, we found that both North and East China have adopted the CM combination of Maxing Shigan Decoction, with the effects of clearing the Lung and relieving asthma. The difference shows that North China uses more heat-clearing medicines, while East China mainly uses dampness-removing medicines.

In conclusion, prescriptions of $\mathrm{CM}$ for the prevention of COVID-19 mainly focus on improving human immunity, while those for treatment of COVID-19 mainly focus on cold-dampness removing and heatclearing, as well as strengthening Spleen. Prescriptions are different in different regions, which may be related to the local climate and geographical environment.

\section{Conflict of Interest}

The authors declared no conflict of interests.

\section{Author Contributions}

Wang YP, Zhang HM, and Lu C conceived this study and developed the first draft of this manuscript. Ji XY and Shi S drafted the manuscript. Liu SH and Tong $L$ collected the data; Shi $S$ created the figures; and $\mathrm{Ji} X Y$ and $\mathrm{Ma} Y$ revised the manuscript. All authors contributed to the editing of the final manuscript and approved the final version.

Electronic Supplementary Material: Supplementary materials (Appendices) are available in the online version of this article at https://doi.org/10.1007/s11655-021-2880-1

\section{REFERENCES}

1. Huang C, Wang Y, Li X, Ren L, Zhao J, Hu Y, et al. Clinical features of patients infected with 2019 novel coronavirus in Wuhan, China. Lancet 2020;395:497-506.

2. Wang C, Horby PW, Hayden FG, Gao GF. A novel coronavirus outbreak of global health concern. Lancet 2020;395:470-473.

3. WHO. https://www.who.int/emergencies/diseases/novelcoronavirus-2019. Accessed on 24 March 2020.

4. Li G, De Clercq E. Therapeutic options for the 2019 novel coronavirus (2019-nCoV). Nature Rev Med Discovery 2020;19:149-150.

5. Wang C, Cao B, Liu QQ, Zou ZQ, Liang ZA, Gu L, et al. Oseltamivir compared with the Chinese traditional therapy maxingshigan-yinqiaosan in the treatment of $\mathrm{H} 1 \mathrm{~N} 1$ influenza: 
a randomized trial. Ann Intern Med 2011;155:217-225.

6. Hsu CH, Hwang KC, Chao CL, Chang SG, Ho MS, Chou $P$. Can herbal medicine assist against avian flu? Learning from the experience of using supplementary treatment with Chinese medicine on SARS or SARS-like infectious disease in 2003. J Altern Complement Med 2006;12:505-506.

7. Yang Y, Islam MS, Wang J, Li Y, Chen X. Traditional Chinese medicine in the treatment of patients infected with 2019-novel coronavirus (SARS-CoV-2): a review and perspective. Int J Biol Sci 2020;16:1708-1717.

8. Huang Z, Fu F, Ye H, Gao H, Tan B, Wang R, et al. Chinese herbal Huo-Gu Formula for treatment of steroidassociated osteonecrosis of femoral head: a 14-years follow-up of convalescent SARS patients. J Orthop Trans 2020. doi: 10.1016/j.jot. [Epub ahead of print]

9. General Office of the State Health Committee, State Administration of Traditional Chinese Medicine Office. Notice of the State on Printing and Distributing the Diagnosis and Treatment Plan of COVID-19 (Trial 7th edition) (EB/OL). (2020-03-03). Http://www.nhc.gov.cn/yzygj/s7653p/202003/4 6c9294a7dfe4cef80dc7f5912eb1989. Shtml.

10. State Council Information Office. State novel Office Held Press Conference: Yu Yanhong Introduced the Important Role of Traditional Chinese Medicine in Participating in COVID-19 Prevention and Treatment (EB/OL). (2020-03-27). http://ghs.saCM.gov.cn/gongzuodongtai/2020-03-27/141

11. Xinhua net. The first Chinese medicine mobile cabin hospital in Wuhan was put into operation.(EB/OL).http://www.xinhuanet. com/politics/2020-02/14/c_1125576187.htm,2020-2-14.

12. State Pharmacopoeia Commission. Pharmacopoeia of the People's Republic of China: Part I . Beijing: China Pharmaceutical Science and Technology Press; 2015:1-1750.

13. Nanjing University of Traditional Chinese Medicine. Dictionary of Traditional Chinese Medicine. 2nd ed. Shanghai: Shanghai Science and Technology Press; 2006:1-2094.

14. Gao XM. Chinese medicine. Beijing: Chinese Medicine Press; 2002:1-631.

15. Wang D, Hu B, Hu C, Zhu F, Liu X, Zhang J, et al. Clinical characteristics of 138 hospitalized patients with 2019 Novel Coronavirus-Infected Pneumonia in Wuhan, China(J/ OL). (2020-02-13). JAMA 2020. https://jamanetwork.com/ journals/jama/fullarticle/2761044.

16. Fan YP, Wang YP, Zhang HM, Wang YY. Analysis on treatment of novel coronavirus pneumonia from cold disease. J Tradit Chin Med (Chin) 2020;61:369-374.

17. Xue BS, Yao KW, Xue YX. Traditional Chinese medicine theory analysis of "Qingfei Paidu Decoction" for rapid and effective treatment of novel coronavirus pneumonia. J Tradit Chin Med (Chin) 2020;61:461-462.

18. Fan YP, Wang YP, Ma Y, Zhao C, Zhang HM. Analysis on composition mechanism of Qingfei Paidu Tang from pathogenesis of cold pestilence of COVID-19 (J/OL). Chin J Exp Tradit Med Formulae (Chin):1-4(2020-04-10). https:// doi-org-s.vpn.ccmu.edu.cn/10.13422/j.cnki.syfjx.20201157.

19. Tong $X L$, Li XY, Zhao LH, Li QW, Yang YY, Lin YQ, et al. Exploration of TCM prevention and treatment strategies of novel coronavirus pneumonia from the perspective of "cold-damp epidemic". J Tradit Chin Med (Chin) 2020;61:465-470, 553.
20. Cinatl J, Morgenstern B, Bauer G, Chandra P, Rabenau H, Doerr HW. Glycyrrhizin, an active component of liquorice roots, and replication of SARS-associated coronavirus. Lancet 2003;361:2045-2046.

21. Du HK, Song FW, Zhou X, Li H, Zhang JP. Effect of amygdlin on type I and III collagen expression in rats with pulmonary fibrosis induced by bleomycin. J Chin PLA Postgrad Med Sch (Chin) 2009;30:712-714+717.

22. Gao XL, Xiong S, Wang YF, Wang XY, Zhang MY, Yuan $Y Q$, et al. Preliminary study on the anti-coxsackievirus B3 effect of three effective parts of patchouli in vitro. Tradit Chin Med Mater (Chin) 2009;32:761-764.

23. Wei XL, Peng C, Wan F. Study on the anti-respiratory virus effect of Patchool in vitro. Pharmacol Clin Chin Mater Med (Chin) 2013;29:26-29.

24. Peng SZ, Li G, Qin Z, Su ZR, Zhang FX, Lai XP. Effect of different extract parts of Pogostemon cablin (Blanco) Benth on in vivo anti-influenza virus. Lishizhen Med Mater Med Res (Chin) 2011;22:2578-2579.

25. Wang C, Cao B, Liu QQ, Zou ZQ, Liang ZA, Li G, et al. Oseltamivir compared with the Chinese traditional therapy maxingshigan-yinqiaosan in the treatment of $\mathrm{H} 1 \mathrm{~N} 1$ influenza: a randomized trial. Ann Intern Med 2011;155:217-225.

26. Liu P, Zhao HP, Luo YM. Anti-aging implications of Astragalus Membranaceus (Huangqi): a well-known Chinese tonic. Aging Dis 2017;8:868-886.

27. Li YK, Li W, Fu CM, Song Y, Fu Q. Lonicerae Japonicae Flos and Lonicerae Flos: a systematic review of ethnopharmacology, phytochemistry and pharmacology. Phytochem Rev 2019;22:1-61.

28. Du CY, Choi RC, Zheng KY, Dong TT, Lau DT, Tsim KW. Yu Ping Feng San, an ancient Chinese herbal decoction containing Astragali Radix, Atractylodis Macrocephalae Rhizoma and Saposhnikoviae Radix, regulates the release of cytokines in murine macrophages. PLoS One 2013;8:e78622.

29. Li YC, Zhang W. Inheritance and exertion of the thought of "Three Factors and Conditions" in the Yellow Emperor's Inner Canon by Shi Bing Lun. Chin J Basic Med Tradit Chin Med (Chin) 2017;23:31-32.

30. Huang LL, Wang JA, Wei Q. Analysis on the law of traditional Chinese medicine for the prevention and treatment of novel corona (J/OL). Chin Med Herbs:1-11(2020-04-21). http://wfs.sxsrsc.com: $8000 /$ rwt/CNKI/http/ NNYHGLUDN3WXTLUPMW4A/kcms/detail/44.1286. R.20200327.1416.002.html.

31. Du S, Peng X, Li F, Yu Z. Analysis on syndrome differentiation and typing of epidemic Japanese encephalitis treated by traditional Chinese medicine in 60 years. Chin $\mathrm{J}$ Basic Med Tradit Chin Med (Chin) 2017;23:31-32.

32. Jiang D, Zhuo QY, Chen XM, Hu J. Enlightenment of diagnosis and treatment of SARS in Beijing and Guangzhou. Lishizhen Med Mater Med Res (Chin) 2017;28:1167-1169.

33. Bai $A J$, Liu XD. Variation characteristics of precipitation in Eastern China in recent 50 years and its relation with drought and flood disasters. J Trop Meteorol (Chin) 2010;26:194-200.

(Accepted August 28, 2020; First Online January 13, 2022) Edited by ZHANG Wen 